Paul H. Mayo

\title{
Critical care ultrasonography: the Italian approach
}

Received: 25 April 2013

Accepted: 26 June 2013

Published online: 9 July 2013

(C) Springer-Verlag Berlin Heidelberg and ESICM 2013

P. H. Mayo (๘)

Hofstra North Shore-LIJ School of Medicine, Hempstead, NY, USA

e-mail: mayosono@gmail.com

Hemodynamic failure is a common problem in the emergency department (ED) and critical care unit. When presented with the hypotensive patient, the intensivist must rapidly establish diagnosis and management strategy. This includes ruling out imminently life-threatening causes for shock, categorization of the type of shock, and prompt initiation of a logical treatment strategy. Is point of care ultrasonography useful in this process?

The answer is a resounding yes. In a recent issue of Intensive Care Medicine, Volpicelli et al. [1] reported on the accuracy of a comprehensive ultrasonography examination in establishing diagnosis in the patient who presents with hypotension in the ED. Their ultrasound results showed a near perfect concordance with those determined by an expert review panel in those cases where the reviewers could determine the cause for the hypotension. The authors are commended for the meticulous methodology of the study and for the importance of their results to the emergency medicine and critical care community.

The concept of a whole body ultrasonography approach to critical illness is not a new one. Lichtenstein and Axler [2] described it in 1993. Lung ultrasonography has been particularly well described [3] with a useful algorithmic approach to respiratory failure [4]. The special attribute of the Volpicelli study is its combination of lung with cardiac ultrasonography for point of care assessment of hypotension. This is a powerful combination, indeed.

Several aspects of the study are particularly noteworthy. The examination took an average of $5 \mathrm{~min}$. For those readers without personal experience in the technique, I assure you that this is an accurate estimate of the time required. The examination was done immediately at the bedside of the patient. Point of care ultrasonography avoids the delay that is inevitable in waiting for the imaging study to be performed, in its interpretation, and in the transmission of the results. There is no clinical disassociation between the offline radiology reader and the clinical reality at the bedside because the frontline clinician immediately integrates the results of the point of care ultrasonography examination with the history, the standard physical examination, and laboratory results. No longer is the imaging study performed in vacuo. Instead, it is fully integrated into the bedside management of the patient by the clinician who is actually at the bedside.

The results of the study are of no surprise to those who use point of care ultrasonography on a regular basis in the ED or intensive care unit. The true worth of the study is that it presents clear evidence to support the concept that point of care ultrasonography should be a routine matter in the evaluation of shock state, with the proviso that it be incorporated into the other mandatory components of the initial evaluation, i.e., history, physical examination, and laboratory assessment. In many ways, point of care ultrasonography is simply an extension of standard physical examination, which is a keystone in the evaluation of any patient.

The question remains, where does the Volpicelli report fit into "the big picture". Why is it that point of care ultrasonography is not the universal standard in critical care medicine? One problem is obvious: the need for training. The Volpicelli team consisted of attending level physicians who had extensive training and experience 
( $>400$ studies per year for at least 5 years). The challenge to the critical care field is how to provide competence in critical care ultrasonography to those physicians who need it. For physicians in training, support comes from the international statement on training in critical care ultrasonography [5]. This document, written with the support of the European Society of Intensive Care Medicine (ESICM), states: "Basic-level critical care echocardiography and general critical care ultrasound should be a required part of the training of every ICU physician."

Trainees need to insist that ultrasonography be a standard part of fellowship training. For those intensivists who have completed training, heavy clinical and personal responsibilities may make it difficult to schedule training. Some attendings may come from an adverse training environment, where their subspecialty colleagues will not help. However, there are several avenues for training. In Europe, the ESICM and other professional societies are developing effective training courses. In North America, the professional societies have well-developed training tracks, most notably the American College of Chest Medicine. Several groups in the USA have developed local training cooperatives, a concept easily adapted for local use. In Australia, there is a well-developed series of courses sponsored by the professional societies.

The only small criticism that I have of the Volpicelli study is that the authors express concern that they do not present evidence that ultrasonography alters outcome or that it is cost-effective. My opinion is that this is a question that will be very difficult to answer, as it is akin to asking whether history and physical examination alters outcome or is cost-effective for the diagnosis and management of the critically ill. Some truths are so selfevident, that we must hold them to be true. This principle applies to point of care ultrasonography for assessment of cardiopulmonary failure. The Volpicelli study shows that ultrasonography works as a diagnostic tool; now the challenge is to train intensivists in its use. The focus of research should be on how to develop training systems that are efficient and effective for both critical care trainees and attending level intensivists.

It is time for critical care clinicians to adopt a new paradigm for imaging, instead of relying on the radiologist and cardiologist to provide the primary ultrasonography service for us. We must step up to do it, interpret it, and apply the results immediately to the urgent clinical question at hand, just as we do with the standard physical examination. We should all aim to be as skilled as the Volpicelli team.

Conflicts of interest On behalf of all authors, the corresponding author states that there is no conflict of interest.

\section{References}

1. Volpicelli G, Lamort A, Tullio M, Cardinale L, Giraudo M, Stefanone V, Boero E, Nazerian P, Pozzi R, Frascisco MF (2013) Point-of-care multi-organ ultrasonography for the evaluation of undifferentiated hypotension in the emergency department. Intensive Care Med 39:1290-1298. doi: 10.1007/s00134-013-2919-7

2. Lichtenstein D, Axler O (1993) Intensive use of general ultrasound in the intensive care unit. Prospective study of 150 consecutive patients. Intensive Care Med 19:353-355
3. Volpicelli G, Elbarbary M, Blaivas M, Lichtenstein DA, Mathis G, Kirkpatrick AW, Melniker L, Gargani L, Noble VE, Via G, Dean A, Tsung JW, Soldati G, Copetti R, Bouhemad B, Reissig A, Agricola E, Rouby JJ, Arbelot C, Liteplo A, Sargsyan A, Silva F, Hoppmann R, Breitkreutz R, Seibel A, Neri L, Storti E, Petrovic T, International Liaison Committee on Lung Ultrasound for International Consensus Conference on Lung Ultrasound (2012) International evidence-based recommendations for point-of-care lung ultrasound. Intensive Care Med 38:577-591
4. Lichtenstein DA, Mezière GA (2008) Relevance of lung ultrasound in the diagnosis of acute respiratory failure: the BLUE protocol. Chest 134:117-125

5. Expert Round Table on Ultrasound in ICU, Cholley B et al (2011) International expert statement on training standards for critical care ultrasonography. Intensive Care Med 37:1077-1083 